\title{
Evaluación de la Concentración del Jugo de Fique (Furcraea spp) para el Control In Vitro de Phytophthora infestans en Plantas de Papa (Solanum tuberosum $L$ )
}

\author{
Ruben D. Solarte y Oswaldo Osorio \\ Universidad de Nariño, Facultad de Ingeniería Agroindustrial. Departamento procesos industriales, \\ Pasto, Colombia. (e-mail: Osorio_oswaldo@udenar.edu.co; Osorio_oswaldo@hotmail.com)
}

Recibido Ene. 16, 2014; Aceptado Mar. 10, 2014; Versión final recibida Abr. 29, 2014

\begin{abstract}
Resumen
Se ha evaluado la concentración del jugo de fique (Furcraea spp) para el control In Vitro de Phytophthora infestans en plantas de papa (Solanum tuberosum $L$ ). Se determinó el contenido final de sapogeninas a las muestras evaluadas de acuerdo al método HPLC-PDA. Para la experimentación se planteó un diseño bifactorial con tres niveles, utilizándose como factores experimentales la temperatura del baño y tiempo de concentración. La evaluación In vitro se realizó a concentraciones de $10000 \mu \mathrm{g} \mathrm{mL}^{-1}$ y para la evaluación se realizó un análisis de varianza a los resultados encontrándose que el tratamiento $\mathrm{C} 1\left(90^{\circ} \mathrm{C} \times 120 \mathrm{~min}\right)$ presenta la mejor combinación para lograr un grado de inhibición del patógeno de $100 \%$. Se concluye que el jugo de fique por su poder anti fúngico se convierte en una alternativa altamente viable para el control de la gota en la papa Phytophthora Infestans.
\end{abstract}

Palabras clave: jugo de fique, extracto, control in vitro, papa, phytophthora infestans.

\section{Evaluation of Fique (Furcraea spp) juice Concentration for the In Vitro Control of Phytophthora infestans in Potato Plants (Solanum tuberosum $L$ ).}

\begin{abstract}
The concentration of fique juice (Furcraea spp) for the In Vitro control Phytophthora infestans in potato plants (Solanum tuberosum $L$ ) has been evaluated. The final content of sapogenins in the samples was determined by HPLC-PDA. For the experiments a bifactorial design with three levels was employed, including the bath temperature and the concentration time as the experimental variables. In vitro evaluation was performed at concentrations of $10000 \mathrm{ug} \mathrm{mL}-1$ and for the assessment analysis of variance of the results was performed finding that the $\mathrm{C} 1$ treatment $\left(90^{\circ} \mathrm{C} \times 120 \mathrm{~min}\right)$ offers the best combination to achieve a pathogen inhibition degree of $100 \%$. It is concluded that fique juice, because of its anti fungal power, repressents a good alternative for control of the "late blight" disease caused by the pathogen Phytophthora infestans.
\end{abstract}

Keywords: fique juice, extract, control in vitro, potato, phytophthora infestans. 


\section{INTRODUCCIÓN}

La gota en la papa o "tizón tardío", ocasionado por el patógeno phytophthora infestans, ha sido uno de los mayores inconvenientes extendidos en los cultivos de papa (Solanum tuberosum L.) sobre todo en zonas húmedas del mundo (Proinpa, 2006). En Colombia se reporta como el limitante de mayor incidencia en el cultivo (Salazar, 2000; Castaño y Castro, 2006; Jaramillo, 2004), debido principalmente a condiciones climáticas para el desarrollo del patógeno y a siembra de materiales altamente susceptibles, como las variedades Diacol Capiro, Parda pastusa, ICA Nevada y Tuquerreña (Jaramillo, 2004). El bajo control sobre el patógeno limita la producción de la papa, puesto que, al no realizarse una adecuada estrategia de manejo, las perdidas pueden oscilar entre el 70 y 100\% de la producción (Cevipapa, 2002).

El manejo de este problema fitosanitario se ha basado en el uso de productos sintéticos, en donde los agricultores realizan aplicaciones calendario con moléculas protectantes como Mancozeb y sistémicos con productos a base de Metalaxil-M [methyl N-(methoxyacetyl)-N-(2,6-xylyl)-D-alaninate] Y Cymoxanil [1-(2ciano-2-metroximinoacetil)-3-etilurea] de manera intensiva (Castaño y Castro, 2006; Martínez y Osorio, 2007). El uso indiscriminado de estos productos actuando en el mismo sitio de acción ha provocado una alta presión de selección y sensibilidad reducida del patógeno (Salazar, 2000; Carreño et al., 2006; Pérez y Forbes, 2008; García et al., 2008), estudios realizados en las regiones IV y V de chile destacan que 254 aislamientos de Phytophthora Infestans se tornaron resistente a Metalaxil (Riveros et al., 2003), sumado a esto la contaminación por su residualidad y acumulación en suelos, plantas, animales y hombre, ha ocasionado alteraciones en los proceso bioquímicos normales y por lo tanto, graves enfermedades (Bravo et al., 2000). Como posible solución a esta problemática algunos autores han reconocido en los extractos de diferentes especies de plantas propiedades fungicidas, cualidades que han generado interés por sus resultados promisorios en laboratorio e invernadero (Castaño y Castro, 2006).Destacando que hay una gran variedad de especies de interés para la investigación, de la cual menos del $10 \%$ de la población vegetal ha sido evaluada en la búsqueda de actividad biológica (Harvey, 2000).

Preciado y Rangel (2006) y Rojas (2008) realizaron aproximaciones en la evaluación del potencial químico que tiene el jugo de fique en el control de Phytophthora Infestans, encontrando que esta especie del genero Furcraea spp. Utilizada principalmente en la extracción de fibras, presenta cualidades fungicidas que pueden ser utilizadas en el manejo de este problema fitosanitario, ya que posee propiedades tensoactivas, plaguicidas por los contenidos de esteroides naturales entre los que están, las saponinas y fitoesteroles (Preciado y Rangel, 2006; Rojas, 2008; Gómez y Vanegas, 2001), componentes que pueden interactuar como limitantes en el crecimiento del patógeno.

Benavides et al.,(2012) realizaron estudios más detallados sobre los contenidos de sapogeninas por HPLC tanto en jugo fresco de fique (Furcraea gigantea) como fermentado, encontrando Hecogenina y Tigogenina; en fresco cuantificaron $101,5 \mathrm{ppm}$ y $90,5 \mathrm{ppm}$ respectivamente y en el jugo fermentado encontraron una relación 6,8/3,2 de estos componentes pero variando de acuerdo al tiempo de fermentación. Es importante anotar que estos componentes son importantes no solo en la industria farmacéutica si no que también son los que pueden actuar como los componentes limitantes del Jugo de fique en un posible Bioinsumo de Fique, por ello la importancia de poder medir y controlar la presencia de estos componentes en el proceso de concentración del jugo de fique.

De la hoja de fique (Furcraea spp.), solo el $4 \%$ del total corresponde a la fibra, el $96 \%$ son desechos de los cuales el $70 \%$ es jugo. (MADR, 2006). Los desechos generados, especialmente los líquidos sin un manejo adecuado ocasionan problemas de contaminación hídrica afectando la ictiofauna de las quebradas y la disponibilidad de recursos hídricos para consumo humano y animal (Mojica y Paredes, 2004). Tras diversas evaluaciones del jugo de fique a nivel in vitro como potencial inhibidor del fitopatógeno Phytophthora infestans causante de la gota en la papa, se concluyo que a concentraciones de $75.000 \mu \mathrm{g} \mathrm{mL}^{-1}$ presenta una inhibición satisfactoria del fitopatogeno (Álvarez et al., 2011). La concentración efectiva reportada por Álvarez et al., (2011) es muy alta para fines de aplicación en campo, situación que dificulta su uso por su alto contenido de agua, por lo tanto concentrar el extracto de fique mediante la evaporación es una posible solución.

La evaporación es una operación unitaria empleada para remover agua de los alimentos líquidos diluidos y obtener un producto líquido concentrado (Singh y Heldman, 1993; Geankoplis, 1998). Entre los beneficios aportados por este proceso está la reducción de las necesidades de espacio para almacenamiento al concentrar los alimentos antes de deshidratarlos, congelarlos o esterilizarlos, ya que se reduce su peso y volumen; la inactivación de las enzimas pectinasas; la protección contra el deterioro microbiano y prolongación de la vida útil de conservación. (Avalo et al., 2009). El objetivo de esta investigación fue evaluar las temperaturas y tiempo adecuados de evaporación de agua mediante un rotaevaporador y posteriormente evaluar in vitro el extracto. 


\section{MATERIALES Y METODOS}

\section{Extracción del jugo de fique}

La materia prima usada en esta investigación fue hojas de fique de la variedad negra común (Furcraea gigantea Vent.), recolectadas en el municipio del Tambó Nariño, Colombia y trasladadas hasta la Planta Piloto de la Universidad de Nariño. Las hojas de fique fueron sometidas a un proceso de limpieza y desinfección con el fin de eliminar todos los residuos contaminantes presentes. Posteriormente se procedió a realizar la extracción del jugo en un molino de rodillos, luego se realizo un filtrado en lienzo y se sometió al jugo a un proceso de fermentación por cuatro días a una temperatura de $35^{\circ} \mathrm{C}$ (Álvarez et al, 2011).

\section{Diseño Experimental}

Se utilizó un diseño bi-factorial con tres niveles, para un total de 9 tratamientos con tres repeticiones cada uno; como testigo se utilizó extracto de fique fermentado.

La realización de los ensayos se llevó a cabo en un rotaevaporador marca EYELA USB 2000 a una presión de vacío de 10 psi y una velocidad de giro del balón de muestra de $30 \mathrm{rpm}$. Mediante estos ensayos se buscaba determinar cuál de los tratamientos a diferentes temperaturas entre $70^{\circ} \mathrm{C}$ y $90^{\circ} \mathrm{C}$ presentaba los mejores resultados de concentración del jugo de fique y posteriormente evaluar su actividad fúngica.

\section{Actividad de agua}

La prueba se realizó en un equipo AquaLab LITE referencia 7614 con un rango de medida de 0,03 a 1 . Para la realización de la prueba se tomo una muestra aproximadamente de 1 gramo, se colocó en una copa y esta a su vez en el equipo, se espero un tiempo aproximado de 3 min y se procedió a realizar la lectura de actividad de agua (Aw).

\section{Evaluación In Vitro}

Para la evaluación in vitro se planteó un nuevo diseño completamente al azar (DCA) en donde se incluyeron dos tratamientos más C10 (Jugo de fique sin concentrar), y C0 (Testigo absoluto - en caja sin enmendar). De cada uno de los tratamientos se evaluó el poder inhibidor sobre el hongo Phytophthora Infestans de los cuales se aplica al medio $10.000 \mu \mathrm{g} \mathrm{mL}-1$ de bioinsumo, dosis recomendada por Álvarez et al (2010). La unidad experimental a evaluar es una caja petri, y cada tratamiento se realizo por triplicado para un total de 33 unidades experimentales.

\section{Sensibilidad del patógeno Phytophthora infestans}

El comportamiento in vitro del patógeno se determinó por medio del crecimiento radial de las colonias de P. infestans, para esto se usaron discos de micelio de $1,2 \mathrm{~cm}$ de diámetro del patógeno, obtenidos con sacabocado, y fueron establecidos en cajas Petri con medio agar tomate enmendado con los diferentes tratamientos; cada unidad experimental fué evaluada mediante el programa gráfico ImageJ (Rasband, 2004) después de un período de incubación de 8 días a temperatura promedio de $18^{\circ} \mathrm{C}$. Se determinó el porcentaje de crecimiento utilizando la relación propuesta por Riveros et al., (2003); así:

$P C=\frac{D M C M-1,1 \mathrm{~cm}}{D M C A} \times 100$

En la ecn. (1), PC es el porcentaje de crecimiento; DMCM es el diámetro medio de colonia creciendo en el tratamiento; $1,2 \mathrm{~cm}$ es el diámetro del cilindro con micelio; y DMCA es el diámetro medio de colonia sin enmendar.

Con base en el porcentaje de crecimiento se determinó la sensibilidad del aislamiento evaluado mediante la escala propuesta por Shattock (1988); en donde:

Sensibles (S): Menor del 10\% del crecimiento del testigo Intermedio (I): Entre 10 y $60 \%$ del crecimiento del testigo Resistente (R): Mayor del $60 \%$ del crecimiento del testigo.

Los datos obtenidos se analizaron mediante un análisis de varianza y prueba de comparación de medias LSD de Fisher a un nivel de significancia del 0,05 mediante el programa estadístico Statgraphics plus 5.1 (2002). 


\section{Análisis de sapogeninas por HPLC-PDA}

Las sapogeninas cristalizadas se disolvieron en Acetonitrilo grado HPLC. Esta solución se purificó mediante SPE C18 para eliminar posibles contaminantes se y filtró con acrodisk de $0.45 \mu \mathrm{m}$ y se inyectó en HPLC. Las sapogeninas fueron analizadas por cromatografía liquida de alta resolución con un (Cromatógrafo Breeze 2 Waters con bomba binaria 1525 y Detector de arreglo de diodos PDA 2998 operado con el software Empower 2,0 (Waters, USA). Columna Analítica: Spherisorb® C8 4.6×100 mm; $5 \mu \mathrm{m}$ id.; Fase Móvil: ACN: H2O (60:40), modo isocrático; Flujo: $0.75 \mathrm{ml} / \mathrm{min}$; Detección: 203nm (Hecogenina); Inyección: $20 \mu \mathrm{L}$ loop; Temperatura: 30ํㅜ; y Diluente: Acetonitrilo y Metanol

Para la cuantificación se utilizó el método de patrón externo utilizando como estándares el acido oleanólico al $98 \%$ y acetato de hecogenina al $90 \%$. La curva de calibración se realizó con cinco concentraciones diferentes y dos inyecciones para cada concentración así: Acido oleanólico 20, 60,80,100 y 120 ppm se leyó a $203 \mathrm{~nm}$ y acetato de hecogenina 10,20, 40,60 y 100 ppm a 235nm. Los valores de $R^{2}$ obtenidos fueron de 0,9979 para acido oleanólico y 0,9984 para acetato de hecogenina, con un \% RSD $<1$.

\section{RESULTADOS Y DISCUSIÓN}

La tabla 1 nos muestra los diferentes tratamientos de concentración, teniendo como resultados los porcentajes de crecimiento del fitopatogeno en cada uno de los tratamientos evaluados, en ella podemos observar que los tratamientos C1,C2 y C8 son los que muestran un mejor comportamiento frente al crecimiento del patógeno, sin embargo estos resultados deben ser analizados mas a profundidad teniendo en cuenta otras respuestas de la presente investigación

Tabla 1. Resultado de los tratamientos

\begin{tabular}{lcccccc}
\hline Tratamiento & $\begin{array}{c}\text { Temperatura } \\
\left({ }^{\circ} \mathrm{C}\right)\end{array}$ & $\begin{array}{c}\text { Tiempo } \\
(\mathrm{min})\end{array}$ & $\begin{array}{c}\text { Crecimiento medio } \\
\text { micelial }(\mathrm{cm})\end{array}$ & $\begin{array}{c}\text { \% de } \\
\text { Crecimiento }\end{array}$ & $\begin{array}{c}\text { Aw } \\
\text { Concentración } \\
(\%)\end{array}$ \\
\hline C0 & Testigo Absoluto & & 5,425 & 100,00 & $-\cdots--$ & --- \\
C1 & 90 & 120 & 1,103 & 0,0 & 0,623 & 92,8 \\
C2 & 90 & 90 & 1,100 & 0,0 & 0,624 & 92,9 \\
C3 & 80 & 90 & 1,252 & 2,8 & 0,701 & 90,4 \\
C4 & 90 & 60 & 1,313 & 4,0 & 0,715 & 87,1 \\
C5 & 70 & 60 & 2,311 & 21,3 & 0,958 & 51,1 \\
C6 & 70 & 90 & 1,426 & 6,2 & 0,909 & 84,5 \\
C7 & 70 & 120 & 1,263 & 2,8 & 0,655 & 90,9 \\
C8 & 80 & 120 & 1,209 & 0,0 & 0,645 & 91,4 \\
C9 & 80 & 60 & 2,264 & 19,2 & 0,915 & 68,2 \\
C10 & Jugó sin concentrar & 2,321 & 20,5 & 0,975 & Sin concentrar \\
\hline
\end{tabular}

Después de concentrado el jugo de fique en cada uno de los tratamientos se determinó estadísticamente mediante un análisis de superficie de respuesta que las variables tiempo y temperatura $(P<0.05)$, presentan diferencias significativa en el proceso de concentración. El análisis presenta un R- cuadrado de $97,6 \%$ y realizando el ajuste por los grados de liberta se establece en $93,5 \%$. Con esto se obtiene que la repuesta óptima para realizar la concentración del jugo de fique fue de $90{ }^{\circ} \mathrm{C}$ x $94,9 \mathrm{~min}$. La Figura 1 muestra la incidencia de la temperatura y el tiempo en el proceso de concentración concluyendo que existe un efecto estadísticamente significativo de estos factores sobre el proceso de concentración $(P<0,05)$. El análisis presenta un R- cuadrado de $97,6 \%$ y realizando el ajuste por los grados de libertad se establece en $93,5 \%$. La repuesta óptima para realizar la concentración del jugo de fique se confirma en $90{ }^{\circ} \mathrm{C} \times 94,9$ min; condiciones en las cuales se obtuvo un $92,9 \%$ de concentración del jugo.

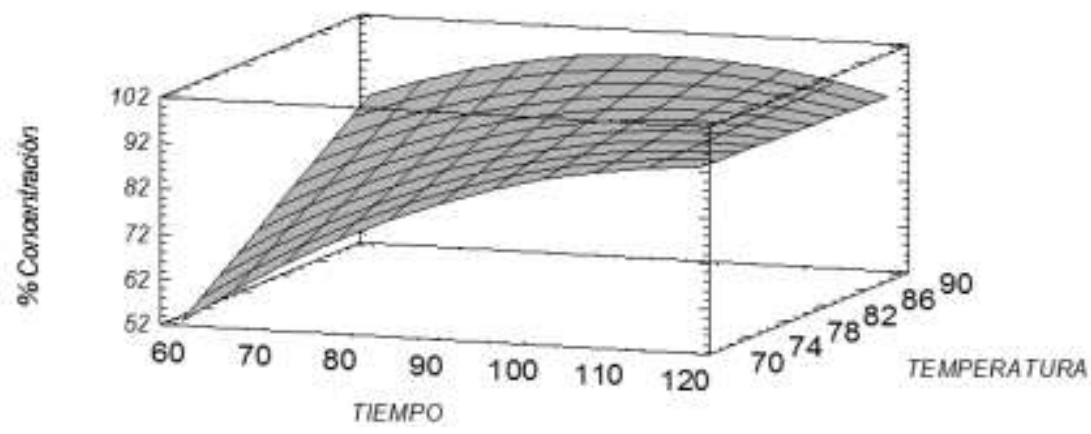

Fig. 1. Superficie de respuesta - porcentaje de concentración 


\section{Actividad de agua}

La actividad de agua presento una reducción considerable en cada uno de los tratamientos; mediante un análisis de varianza se determinó que las variables analizadas tiempo y temperatura inciden en la actividad de agua del jugo de fique concentrado ( $\mathrm{P}$ - Valor $<0.05$ ), siendo el tiempo el más representativo, igualmente se realizo una comparación de muestras entre los tratamientos incluido el tratamiento $\mathrm{C} 10$. Los resultados mostraron que existen diferencias significativas ( $P$-Valor < 0.05 ) a un nivel de confianza del 95\%; al considerar que existe diferencia significativa se realiza una prueba de rangos múltiples mediante el método de Duncan a un nivel de confianza del $95 \%$, con el cual se determina que la media más pequeña es la de los tratamientos $\mathrm{C} 1$ y C2 tal como se muestra en la figura 2. En esta figura, las letras iguales indican que no hay diferencia estadísticamente significativa (nivel de confianza 95\%)

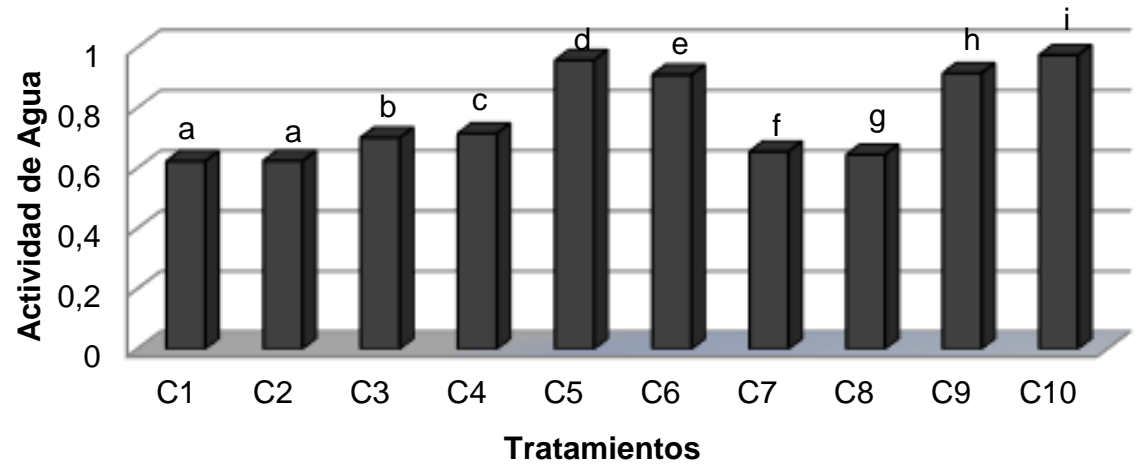

Fig. 2. Actividad de agua en los tratamientos después del proceso de concentración

La actividad de agua se convierte en un factor determinante al momento de considerar la vida útil de un producto, en tal sentido los tratamientos $\mathrm{C} 1$ y $\mathrm{C} 2$ se consideran como los mejores por su baja actividad de agua presentada y control sobre el crecimiento micelear del hongo en estudio, aunque es necesario aclarar que de los dos tratamientos mencionados el segundo presenta las mejores condiciones de manipulación en el proceso, por consiguiente el tratamiento $\mathrm{C} 2$ se determina como el mejor para ser considerado en una evaluación de campo.

\section{Evaluación In vitro}

Analizando el porcentaje de crecimiento in vitro de cada uno de los tratamientos mediante la metodología de superficie de respuesta, se encontró que la variable más influyente en el porcentaje de crecimiento de Phytophthora, es el tiempo $(\mathrm{P}<0.05)$ con un $\mathrm{R}$ - cuadrado de $93,6 \%$, mostrado buen ajuste de los datos; por otro lado con el fin de determinar cuál de los tratamientos evaluados presenta un mejor desempeño en la inhibición del patógeno se realizó un análisis de varianza, con lo cual se determinó que existen diferencias estadísticamente significativas $(P<0.05)$ entre los tratamientos, posteriormente se realizó la comparación de medias mediante la prueba de Duncan, dando como resultados que los tratamientos $\mathrm{C} 1$ y $\mathrm{C} 2$ presentan el mayor poder de inhibición frente al hongo,

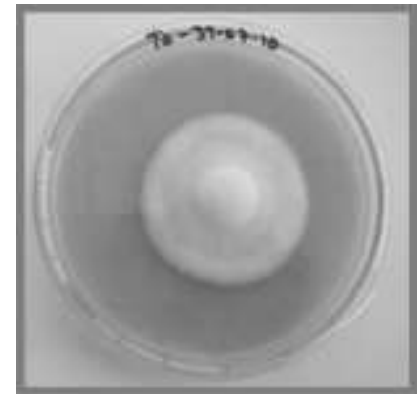

Testigo Absoluto CO

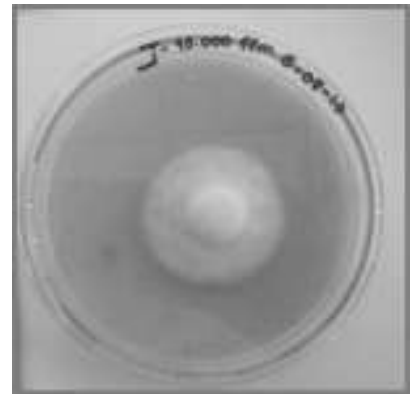

Jugo de fique liquido $\mathrm{C} 1$

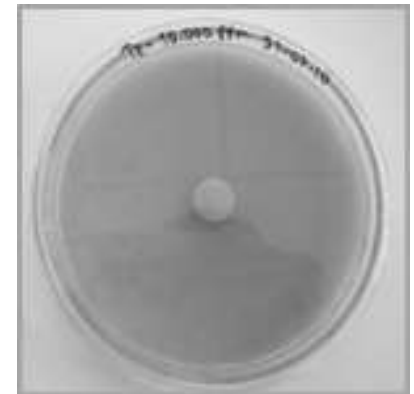

Jugo de fique concentrado C2

Fig. 3: Evaluación in Vitro del jugo de fique

En cuanto a la evaluación in vitro del jugo de fique concentrado (Furcraea gigantea) a una aplicación de $10.000 \mu \mathrm{g} \mathrm{mL}-1$ se demostró que todos los tratamientos tienen efecto en la inhibición del patógeno $P$. Infestans (Figura 4). En la figura 4, las letras iguales indican que no hay diferencia estadísticamente significativa (nivel de confianza 95\%). Según la escala de Shattock los tratamientos C1, C2, C3, C4, C6, C7, C8 presentan crecimientos por debajo del 10\% indicando un nivel de sensibilidad alto; los otros tratamientos 
(C5, C9 y C10) presentan nivel intermedio en la escala. El efecto fúngico del jugo de fique concentrado con respecto al liquido, puede deberse a la concentración de los metabolitos secundarios presentes, especialmente sapogeninas, las cuales han sido reportadas en este sentido por (MADR \& MAVDT, 2006); como insumos genéricos de alta actividad biológica y especificidad.

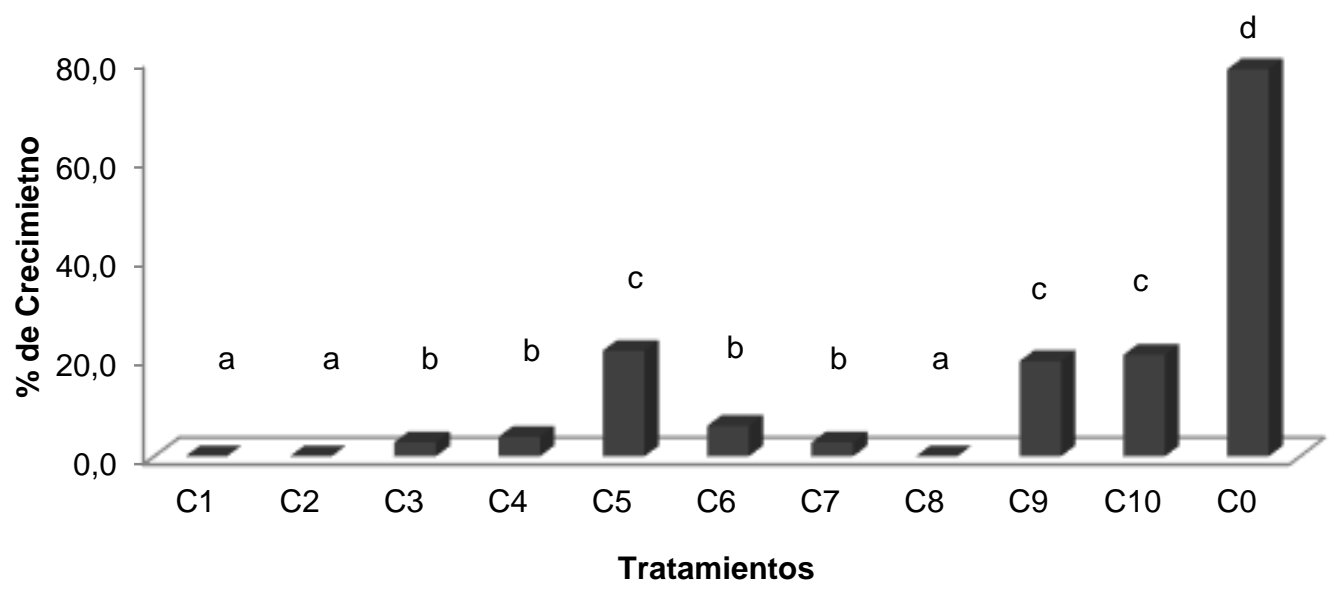

Fig. 4. Porcentaje de crecimiento radial a nivel in vitro en cada uno de los tratamientos

La evaporación del agua con el rotaevaporador tuvo una gran capacidad de concentración de todos los componentes presentes en el jugo de fique, lo cual permitió prolongar la conservación del jugo. El jugo de fique concentrado presenta una excelente capacidad de inhibición del fitopatogeno Phytophthora Infestans manteniendo muchas de sus propiedades después de la aplicación de cada uno de los tratamientos de concentración, sin embargo a pesar de todas sus bondades inhibitorias, el jugo de fique concentrado en los tratamientos más fuertes $(\mathrm{C} 1, \mathrm{C} 2$ y $\mathrm{C} 8)$ presenta una consistencia viscosa lo que dificulta su manejo, sin embargo el tratamiento $\mathrm{C} 7$ es efectivo y también presenta buenas condiciones de manejo para posteriores evaluaciones en campo.

\section{Contenido de saponinas en el bioinsumo de fique}

En la tabla 2 se muestra el comportamiento con respecto al contenido de Hecogenina de las muestras evaluadas, las cuales corresponden a los mejores tratamientos de inhibición sobre el fitopatogeno evaluado. De acuerdo a la figura 4, se puede observar que aún cuando las cantidades de hecogenina son menores en el extracto seco en un $21 \%$, podemos inferir que ese porcentaje presente que asciende a un $79 \%$ y que permanece en el extracto es suficiente para actuar como inhibidor de los procesos de crecimiento de hongos fitopatogenos como lo es Phytophthora Infestans que ataca los cultivos de papa, hongo que fue evaluado inicialmente a nivel in vitro.

Tabla 2. Resultados para Hecogenina en el jugo de fique mediante HPLC

\begin{tabular}{lccc}
\hline & \multicolumn{2}{c}{ Contenido de Hecogenina } \\
\hline & Jugo Fresco & Fermentado 4 días & Concentrado a vacío \\
Área & 539826 & 426725 & 440190 \\
Sobre extracto seco $(\mathrm{mg} / \mathrm{L})$ & 21,64 & 16,79 & 17,37 \\
Sobre Muestra Inicial $(\mathrm{mg} / \mathrm{L})$ & 64,72 & 33,07 & 72,62 \\
\hline
\end{tabular}

El objetivo inicial de la presente investigación tenía como finalidad eliminar el contenido de agua del producto ya fuera fresco o fermentado, y que sus componentes mayoritarios, que se suponían realizaban el efecto inhibidor del hongo, estuvieran allí después de los procesos de concentración. El contenido de Hecogenina comprobado por HPLC en el jugo fresco nos determino su presencia aproximadamente a los 5 min de tiempo de retención; la presencia de dicho componente en el jugo fermentado y el jugo concentrado reporto su presencia en las mismas condiciones de medición por HPLC; esto nos hace pensar que el efecto inhibidor de este bioinsumo puede estar generado por los contenidos de sapogeninas (hecogenina 0 tigogenina) componentes que habían sido reportados como potenciales inhibidores de hongos patógenos y en particular de Phytoptora Infestans, según lo reportado por Álvarez et al. (2011).

Las figuras 5,6 y 7 antes mencionadas hacen referencia a los cromatogramas del HPLC, donde a un tiempo de corrido de 5 minutos se evidencian los picos con contenido hecogenina en cada una de las tres muestras. 


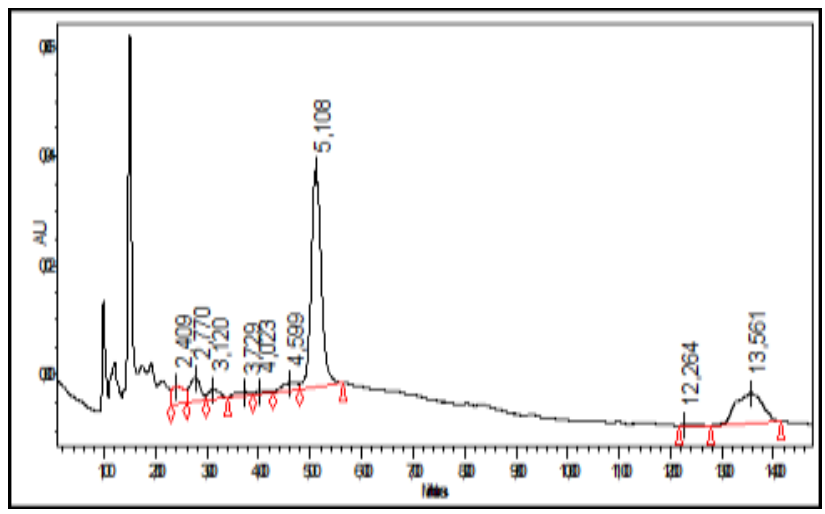

Fig. 5. Cromatograma del contenido de Hecogenina en el jugo de fique fresco.

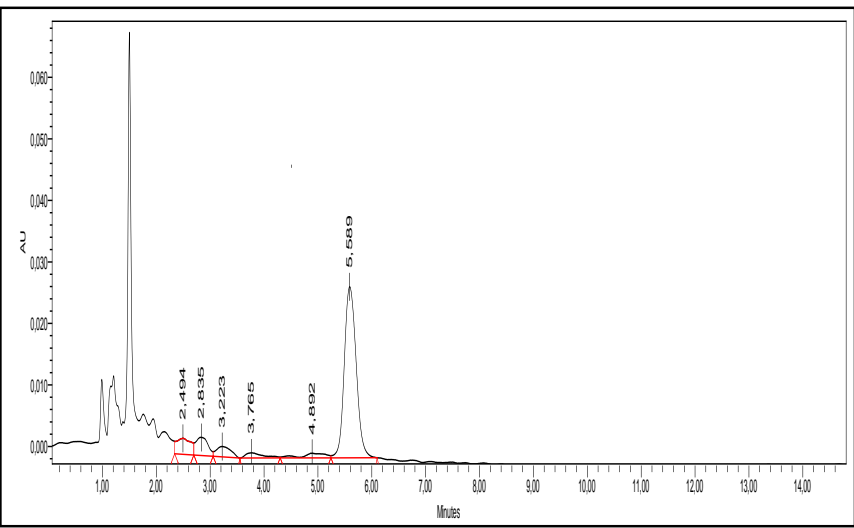

Fig. 6. Cromatograma del contenido de Hecogenina en el jugo de fique fermentado sin concentrar

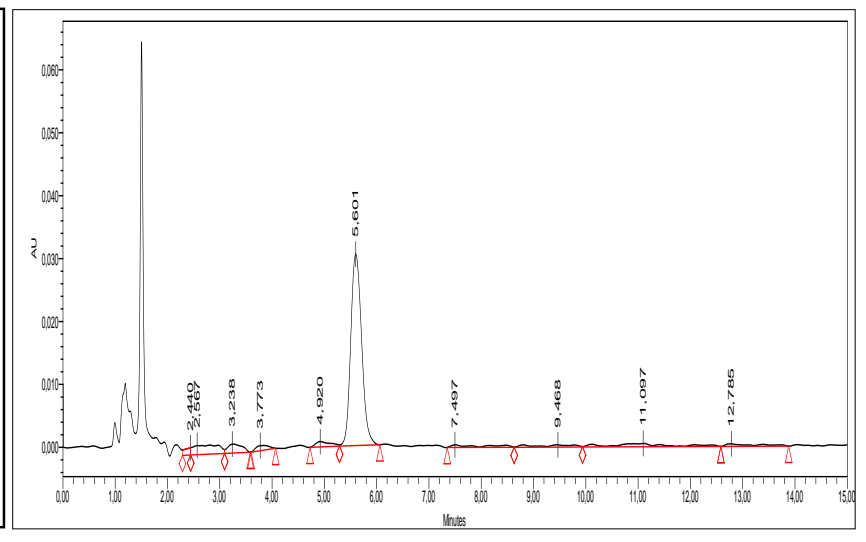

Fig. 7. Cromatograma del contenido de Hecogenina en jugo de fique fermentado concentrado.

\section{CONCLUSIONES}

Basdo en este estudio se obtienen las siguientes conclusiones principales: i) La temperatura y el tiempo son factores que influyen directamente en la concentración del jugo de fique e indirectamente en la actividad de agua; ii) Mediante la concentración se redujo la actividad de agua e inversamente aumento el periodo de vida útil del jugo de fique; iii) Los componentes del jugo que han sido reportados con capacidad antifungica, (sapogeninas) se mantuvieron en un $79 \%$ en el proceso de concentración del jugo fermentado; iv) Los tratamientos con mayor poder de inhibición son el tratamiento $\mathrm{C} 1\left(90^{\circ} \mathrm{C} \times 120 \mathrm{~min}\right)$ y $\mathrm{C} 2\left(90^{\circ} \mathrm{C} \times 90 \mathrm{~min}\right)$; y v) El jugo de fique por su poder anti fúngico se convierte en una alternativa altamente viable a nivel in vitro para el control de la gota en la papa Phytophthora Infestans (Mont.) de Bary.

\section{AGRADECIMIENTOS}

A la Vicerrectoria de Investigaciones de la Universidad de Nariño por su aporte económico para el desarrollo de la presente investigación y al grupo de investigación Tecnologías Emergentes en Agroindustria.

\section{REFERENCIAS}

Alvarez, D. y otros 3 autores. Sensibilidad in vitro de Phytophthora infestans (Mont.) de Bary al extracto de fique (Furcraea gigantea Vent.) y fungicidas sistémicos. Biotecnologia en sector agropecuario y agroindustrial, 9(2), 96-104, (2011).

Avalo, B., S. Perez y M. Tovar. Caracterización preliminar del proceso de concentración del jugo natural de naranja en un evaporador de tres efectos. INCI, ISSN 0378-1844, 34(11), 784-790, (2009).

Benavides, O. L y otros 3 autores. Cuantificación de Sapogeninas del Jugo Fresco y Fermentado de Fique (Furcraea gigantea) mediante Cromatografía Liquida de Alta Resolución (HPLC-PDA). Información Tecnológica, 23(3), 67-76, (2012).

Bravo, L., T. Bermúdez y B. Montes. Inhibición de Fusarium moniliforme mediante polvos vegetales y algunos de sus componentes químicos. Manejo Integrado de Plagas, 57(1), 29-34, (2000). 
Carreño, N. y otros 3 autores. Problemas Fitopatologicos en especies de la familia solanaceae causadas por los género Phytophthora, Alternaria y Ralstonia en Colombia. Agronomía Colombiana, 25(1), (2006).

Castaño, J y R. Castro. Efecto de once extractos vegetales sobre el tizón tardío causado por Phytophthora infestans (Mont de Bary) en papa (Solanum phureja Juz. et Buk), 10(1), 148, (2006).

Cevipapa. El correo de la papa: Evaluación de dos clones de papa con resistencia a Phytophthora infestans como estrategia para el manejo integrado de la gota. Bogota.22p.(2002).

Garcia, M. M., S. Jaramillo y J. Cotes. Sensibilidad de aislamientos colombianos de Phytophthora infestans a cuatro fungicidas sistémicos. Agronomía Colombiana, 26 (1), 47-57, (2008).

Geankoplis, C. Procesos de Transporte y Operaciones Unitarias, $3^{a}$ edición, 1007, Continental. México, (1998).

Gómez, M y E. Vanegas. Evaluación de la producción de esteroides a partir del jugo de fique con Cunninghamella spp. Tesis pregrado, Univ. Pontificia Bolivariana de Medellín, Fac. de Ingeniería Química, Medellin, Colombia (2001).

Harvey, A. Strategies for discovering drugs from previously unexplored natural products (2000) [On line] Drug Discovery Today 5(7): 294-300 (2010).

Jaramillo, S. Monografía sobre Phytophthora infestans (Mont) de Bary. Universidad Nacional de Colombia, Facultad de Ciencias Agropecuarias, Medellin, Colombia (2004).

Martinez, E y J. Osorio. Estudios preliminares para la producción de un biosurfactante bacteriano activo contra Phytophthora infestans (Mont.) De Bary, (2007), Online:http://www.corpoica.gov.co/SitioWeb/ Archivos/Revista/1.Estudiospreliminares.pdf; Consultado: febrero (2010).

Ministerio de Agricultura y Desarrollo Rural (MADR). Guia ambiental del subsector Fiquero, $2^{\text {da }}$ Edición, 2728, Bogota, (2006).

Ministerio de Agricultura y Desarrollo Rural y Ministerio de Ambiente, Vivienda y Desarrollo Territorial. Guía Ambiental del Subsector Fiquero., $2^{\text {da }}$ Edición, Panamericana Formas e Impresos Bogotá, (2006).

Mojica, A y J. Paredes. El cultivo del fique en el departamento de Santander. Centro Regional de Estudios Económicos, Bucaramanga (Santander, Colombia) 154, (2004).

Peréz, W y G, Forbes. Manual técnico: El tizón tardío de la papa. Perú, Centro internacional de la papa (CIP), 41, (2008).

Preciado, D y E. Rangel. Extracción de un biofungicida a partir del jugo de fique (Furcraea spp). Trabajo de pregrado, Facultad de ingeniería química, Universidad pontificia bolivariana, Medellín, Colombia, 78, (2006).

Proinpa. Papa andina: Innovación para el desarrollo de los andes 2002-2006. En: http://www.papandina.cip.cgiar.org/fileadmin/documentpool/Administrativo/Informe/Fse/06-10-11Compendio_2002_2006.pdf. Acceso: 20 de Agosto (2010).

Rasband, W.S. ImageJ, National Institutes of Health, Bethesda, Maryland,USA,http://rsb.info.nih.gov/ij/. 1997-2004.

Riveros, F. B y otros 3 autores. Reistencia de Phytophthora infestans (Montagne) de Bary a Metalaxyl en cultivo de papas en el norte de chile. Agricultura técnica Chile, ISSN 0365-2807, 63 (2), 117-124, (2003).

Rojas, M. Características fisicoquímicas del jugo de fique (Furcraea spp.) elaboración y evaluación de un biofungicida útil en el control agroecológico de la gota (Phytophthora infestans) en la papa, Tesis pregrado, Universidad de Nariño, San Juan de Pasto, Colombia, 103. (2008).

Salazar, C. Dinámica temporal de las poblaciones de Phytophthora infestans a los fungicidas Metalaxyl, Cymoxanil y Propamocarb en cultivos de papa, Tesis de maestría, Facultad de Agronomía. Universidad Nacional de Colombia, Bogota, Colombia, (2000).

Shattock, R. Studies on the inheritance o resistance to metalaxyl in Phytophthora infestans. Plant Pathology, 4-11, (1988).

Singh R, y D. Heldman. Introduction to Food Engineering, $2^{a}$ edición, Academic Press, Nueva York, EEUU. 499,(1993).

Statgraphics plus 5.1. Statistical Graphics Corp, (2002). 